\title{
Original
}

\section{Significados culturales del concepto de salud en el trabajo en profesionales en formación}

\section{Cultural meanings of the concept of health at work in professionals in training}

\section{María de los Ángeles Aguilera Velasco', José de Jesús Pérez Solís², Blanca Elizabeth Pozos Radillo³, Martín Acosta Fernández ${ }^{1}$}

1. Instituto de Investigación en Salud Ocupacional. Departamento de Salud Pública. Centro Universitario de Ciencias de la Salud. Universidad de Guadalajara (México)

2. Maestría en Ciencias de la Salud en el Trabajo. Universidad de Guadalajara (México)

3. Departamento de Salud Pública. Centro Universitario de Ciencias de la Salud. Universidad de Guadalajara (México)

Recibido: 21-06-12

Aceptado: 20-07-12

\section{Correspondencia}

María de los Ángeles Aguilera Velasco

Instituto de Investigación en Salud Ocupacional de la Universidad de Guadalajara

Colonia Indendencia

Sierra Mojada 950 Puerta 1 Edificio N Planta Alta

44340 Guadalajara (México)

Tfno: +5233 10585200 ext. 33898

E-mail: aaguileracd@hotmail.com

Resumen

Introducción: El concepto de salud en el trabajo implica una serie de significados para los profesionales de la salud ocupacional, quienes los utilizan para orientar, interactuar, discutir, definir, categorizar e interpretar sus prácticas. Conocer dichos significados es relevan te porque permite aportar datos útiles para el diseño de programas educativos tendientes a la prevención de accidentes y enfermedades laborales.

Objetivo: Explorar los significados culturales del concepto de salud en el trabajo, antes y después de un diplomado con duración de seis meses, en profesionales de la salud ocupacional.

Metodología: Estudio cualitativo con diseño descriptivo exploratorio. Realizado en Guadalajara, México de febrero a julio de 2011. Los datos fueron recolectados con la técnica de listados libres y analizados a través del modelo de dominios culturales.

Resultados: El concepto de salud en el trabajo, antes del diplomado se definió a través de los términos riesgo y seguridad. Después del diplomado, la salud en el trabajo se definió a través de los elementos seguridad y prevención. Conclusiones: La cultura de la prevención se logra a través de un proceso de aprendizaje que incluye conocimientos sobre los riesgos laborales y la normatividad vigente.

Med Segur Trab (Internet) 2012; 58 (228) 224-236

Palabras Clave: Cultura, salud laboral, estudios de intervención. 


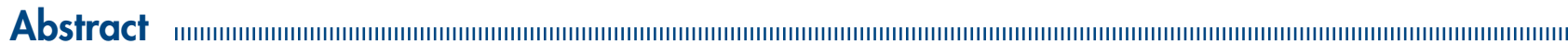

Introduction: The concept of health in the workplace involves a number of meanings for occupational health professionals, who are used to guide, interact, discuss, define, categorize, and interpret their practices. Knowing these meanings is relevate because it allows to provide useful data for the design of educational programmes aimed at the prevention of accidents and occupational diseases.

Objective: Explore the cultural meanings of the concept of health at work, before and after the training with duration of six months, in occupational health professionals.

Methodology: Qualitative study with exploratory descriptive design, in Guadalajara, Mexico from February to July 2011. The data were collected with the technique of free lists and analyzed through the model of cultural domains. Results: The concept of occupational health, before the training was defined through the terms risk and security. After the training, health in the workplace was defined through the elements safety and prevention.

Conclusions: The culture of prevention is achieved through a learning process that includes knowledge of occupational hazards and the existing regulations.

Med Segur Trab (Internet) 2012; 58 (228) 224-236

Key Words: Culture, occupational health, intervention studies. 


\section{INTRODUCCIÓN}

La necesidad de la formación de recursos humanos en salud ocupacional se estable en la Estrategia Mundial de la Salud Ocupacional para Todos", donde se señala que "las actividades de salud ocupacional solo pueden ser competentes si las desempeña personal bien adiestrado". Además se reconoce que en los países en desarrollo hay una gran escasez de expertos y las oportunidades de empleo son mínimas, tanto por la falta de legislación propia, como por la ausencia de programas de formación elaborados por instituciones de adiestramiento vocacional y universidades. Concluyendo que la capacitación de un número suficiente de expertos es otro asunto más del cual tienen que ocuparse los programas nacionales, junto con el establecimiento de medios para que los empleadores, gerentes y los propios trabajadores tengan un mínimo de conocimientos sobre el tema.

Posteriormente, en el Plan Regional OPS/OMS para la Salud de los Trabajadores, se asienta que "formar recursos bumanos para informar y capacitar a trabajadores $y$ empleadores es un reto fundamental y un medio importante para sensibilizar a la dirigencia política y social acerca de la situación preocupante que caracteriza los perfiles de las Salud de los Trabajadores" 2 .

Aunado a lo anterior, la 60. ${ }^{\text {a }}$ Asamblea Mundial de la Salud 2008-2017, a través del documento Salud de los trabajadores: plan de acción mundial, insta a los Estados miembros "a que tomen medidas orientadas a establecer y fortalecer las capacidades institucionales fundamentales, así como la capacidad en materia de recursos bumanos para atender las necesidades sanitarias especiales de las poblaciones activas, obtengan datos fehacientes relativos a la salud de los trabajadores, y los traduzcan en políticas y acciones" 3

No obstante, uno de los problemas más acuciantes todavía, es la falta de fomento de estudios en materia de ciencias de la salud ambiental y ocupacional, que permitan anticipadamente evaluar, identificar y controlar los riesgos. Los servicios de salud y seguridad en el trabajo requieren de una estructura macro, similar a la de las universidades, para ampliar la oferta, capacitar a los distintos actores, aportar conocimientos, formación técnica y colaborar con el movimiento sindical en integrar e incluir el saber de los trabajadores ${ }^{4}$.

En ese sentido, algunas instituciones educativas de países latinoamericanos realizan, desde hace más de una década, importantes acciones de formación de recursos humanos en salud ocupacional que incluyen cursos de pregrado sobre salud en el trabajo sobretodo en la licenciatura de medicina; carreras técnicas para formar profesionales de la salud ocupacional; diversas maestrías en salud en el trabajo; un par de doctorados y abundantes diplomados, tanto desde el ámbito formal como no formal, como en el caso de las asociaciones o federaciones de profesionales de la salud en el trabajo.

Los diplomados se ofrecen a los egresados de licenciatura y son una buena opción para aquellos profesionistas que desean emplearse o actualizarse en el ámbito de la salud ocupacional tanto a nivel empresarial como institucional. Generalmente son programas de formación que tienen una duración máxima de seis meses y una carga horaria semanal no mayor a 10 horas, con la participación de diversos ponentes expertos en la materia.

El diplomado en salud, seguridad en el trabajo y protección al ambiente, en Guadalajara, México, al 2011 se había ofrecido en 39 ocasiones. Es un programa compuesto de nueve módulos que incluyen el estudio sobre la legislación laboral, seguridad en el trabajo, diagnóstico y diseño de programas de seguridad e higiene, metodología de la investigación, programas de cómputo aplicados, educación en ambientes laborales, higiene en el trabajo y protección al ambiente. Participan como formadores, algunos expertos del Instituto Mexicano del Seguro Social (IMSS), de la Secretaria de Trabajo y Previsión Social (STPS) y profesores de diversos centros universitarios y departamentos de la Universidad de Guadalajara. Al finalizar el diplomado, algunos egresados continúan preparándose a través de los programas de maestría y doctorado en salud en el trabajo de la misma institución. En dicho programa a través de tres exámenes (diagnóstico, 
parcial y final) y de tres trabajos finales (diagnóstico de seguridad e higiene, programa de investigación e higiene y proyecto de investigación) se verifican los conocimientos, habilidades y capacidades adquiridas.

Volviendo al tema de interés, además de la falta de fomento de estudios en materia de ciencias de la salud ambiental y ocupacional, también se requiere de investigación sobre educación en salud ocupacional, ya que ésta es prácticamente nula. Dicha problemática es comprensible ante la falta de programas de formación. Sin embargo, los beneficios de llevarla a cabo redundarían en la calidad de mejores eventos de capacitación.

La investigación sobre educación, a través de las ciencias de la educación, estudia los hechos y las situaciones educativas con una perspectiva macroscópica; se refiere a estudios científicos explicativos o comprensivos de fenómenos relacionados con la educación y que son abordados por otras ciencias y disciplinas como la filosofía, la antropología, la economía, la administración, la sociología y, por supuesto, la psicología, desde su mirada particular (no pedagógica), disciplinas y ciencias que aportan conceptos, teorías e instrumentos que apoyan el análisis de problemas de la educación y ofrecen marcos conceptuales a la investigación en educación. Es investigación sobre educación, la que desde el punto de vista de otras disciplinas, realiza contribuciones incidentales a la empresa educativa ${ }^{5}$.

La antropología cognitiva es considerada como una tradición cualitativa de investigación social en estudios sobre educación. La cultura es vista por esta tradición como aquellas organizaciones cognoscitivas o representaciones de los fenómenos materiales. Los patrones culturales se reflejan en el lenguaje, especialmente en la semántica para realizar estudios sobre la cultura del sexo y su papel en las escuelas; epistemologías de los docentes o sus imágenes sobre el conocimiento y sobre su pensamiento pedagógico; los juegos en los descansos escolares; las jergas estudiantiles con respecto a directores, maestros, eventos escolares; culturas de barras; cultura sexual en la escuela, entre otros. Los tipos de análisis han adquirido rica variedad: análisis de escenas y su organización; análisis taxonómico de los términos de un dominio o campo cultural; análisis de contrastes y análisis temático 5 .

En México, en el campo de los estudios sobre educación se han explorado las dimensiones culturales del concepto de tutoría académica en docentes y alumnos de doctorado $^{6}$. En el campo de la salud, la antropología cognitiva ha fundamentado estudios socioculturales enfocados en conocer e incorporar la cultura del usuario en el proceso de atención de salud, a través de las cuales ha sido posible comprender, por ejemplo, los elementos para la construcción de las bases conceptuales de la salud intercultural ${ }^{7}$; las nociones de salud, enfermedad y muerte ${ }^{8,9}$; el punto de vista de la madres en el proceso de atención primaria infantil ${ }^{10}$; las dimensiones conceptuales del bienestar en personas con padecimientos crónicos ${ }^{11} \mathrm{y}$ de la salud en personas jubiladas ${ }^{12}$; perspectivas para el no consumo de drogas en alumnos de bachillerato ${ }^{13}$ así como la descripción de los conocimientos sobre los factores humanos que causan accidentes en la industria arnesera ${ }^{14}$.

Respecto del concepto de salud en el trabajo, la Organización Internacional del Trabajo (OIT) y la Organización Mundial de la Salud (OMS) establecieron desde 1950, y actualizaron en 1995, a través del Comité Conjunto de Salud Ocupacional OIT/OMS, que "la finalidad de la salud en el trabajo consiste en lograr la promoción y el mantenimiento del más alto grado de bienestar físico, mental y social de los trabajadores en todos los trabajos; prevenir todo daño causado a la salud de éstos por las condiciones de su trabajo; protegerlos en su empleo contra los riesgos resultantes de agentes perjudiciales a su salud; colocar y mantener al trabajador en un empleo adecuado a sus aptitudes fisiológicas y psicológicas; y en suma adaptar el trabajo al hombre y cada hombre a su actividad" 15 .

La práctica de la salud laboral, según García, Ruiz y García ${ }^{16}$, surge como respuesta al conflicto entre salud y condiciones de trabajo, y se ocupa de la vigilancia e intervención sobre las condiciones de trabajo y la salud de los trabajadores. Sus principales objetivos son "identificar, eliminar o modificar los factores relacionados con el trabajo que ejerzan un efecto perjudicial para la salud del trabajador; potenciar aquellos con un efecto beneficioso para su salud y bienestar; y considerar el tratamiento y la rehabilitación del trabajador que ha sufrido un problema de salud". 
El Código de Ética de los Profesionales de Salud en el Trabajo, elaborado por la Comisión Internacional de Salud Ocupacional (CISO) en el 2002, establece que el propósito de la salud ocupacional es servir a la salud y el bienestar social de los trabajadores en forma individual y colectiva así como contribuir además al mejoramiento de la salud pública y del medio ambiente ${ }^{17}$.

En el código se define a los profesionales de salud ocupacional como a todos aquellos que, en el ejercicio de su profesión, desempeñan tareas, proveen servicios o están involucrados en una práctica de seguridad y salud ocupacional. Y se indica que existe una amplia gama de disciplinas relacionadas con la salud ocupacional, con tendencia a utilizar las competencias de los profesionales de la salud ocupacional con enfoque de equipo multidisciplinario. En cuanto a la formación de los profesionales de la salud ocupacional, el código establece que éstos deben adquirir y mantener la competencia necesaria para ejercer sus obligaciones ${ }^{17}$.

En el código también se señalan los derechos y las obligaciones de los profesionales de la salud ocupacional, entre los que destacan el desarrollo de organizaciones y culturas de trabajo que promuevan ambientes sociales positivos y apoyen la productividad de los procesos. En ese contexto, el concepto de cultura de trabajo pretende significar una reflexión sobre los sistemas de valor esencial adoptados por las tareas emprendidas. En la práctica, la cultura se refleja en sistemas de administración, las políticas de personal, el principio de participación, las políticas de entrenamiento, y la gestión de calidad de lo emprendido $^{17}$.

La Estrategia Comunitaria de Salud y Seguridad en el Trabajo 2002-2006 propone facilitar la aplicación de la legislación existente en Europa en materia de salud y seguridad en el trabajo. Indica que deben cumplirse tres exigencias para garantizar un entorno de trabajo seguro y saludable: la consolidación de la cultura de prevención de los riesgos, una mejor aplicación del derecho existente y un planteamiento global del bienestar en el trabajo. Para satisfacer esas condiciones, la estrategia comunitaria propone tres grandes orientaciones: la adaptación del marco jurídico, el apoyo a las gestiones de progreso (elaboración de mejores prácticas, diálogo social y responsabilidad social de las empresas) y la integración de la problemática de la seguridad y la salud en el lugar de trabajo en otras políticas comunitarias ${ }^{18}$.

Posteriormente, a través de la Conferencia Internacional de presentación de la Estrategia Comunitaria de Seguridad y Salud en el Trabajo 2002-2006, se señala que "la cultura de la prevención sólo puede darse como el resultado de un proceso de aprendizaje que debe dar comienzo en la enseñanza primaria. En este nivel formativo el aprendizaje debe integrarse en la formación en valores, concretamente dentro del valor salud. Como en toda enseñanza de valores, la metodología ha de ser de carácter transversal y debe presentar los riesgos de la propia escuela como un primer ejemplo de riesgos laborales. Este proceso formativo ba de continuarse en la formación profesional, mediante la integración de las actividades preventivas en el propio proceso formativo curricular, $y$ especialmente en sus aspectos prácticos. El concepto sería: un trabajo está bien hecho si se hace de manera segura. Este mismo concepto debería centrar la formación preventiva en los niveles superiores del sistema educativo, si bien en este caso la atención debería dirigirse no tanto al alumnado, como posible receptor del daño, sino más bien hacia las personas que puedan encontrarse bajo su mando. Las funciones desempeñadas por estos futuros profesionales será clave en este sentido".

Al respecto, desde hace más de 10 años existe en España un marco legal que presenta la cultura de la prevención como el eje, el objetivo y el medio fundamental para conseguir una mejora efectiva de las condiciones de trabajo. Donde se busca que la prevención se integre en el día a día como un auténtico estilo de vida y no como una imposición ${ }^{19}$.

Por otro lado, Gómez ${ }^{20}$ indica la salud ocupacional es entendida como la salud del trabajador en su ambiente de trabajo, pero que sin embargo, el concepto de salud en el trabajo ha tenido importantes cambios conceptuales y metodológicos en los últimos años y que se han desarrollado valiosas experiencias que ponen de relieve la importancia de la 
salud de los trabajadores para el desarrollo de los países. Indica que el concepto de salud ocupacional tiende a sustituirse por el concepto de salud de los trabajadores, porque es más amplio y comprende además de la salud ocupacional, la salud del trabajador fuera de su ambiente laboral. Incluye los accidentes de trabajo, las enfermedades ocupacionales, las patologías asociadas al trabajo y las derivadas de su vida fuera de su centro de trabajo.

A pesar de lo anterior, se considera que el concepto de salud en el trabajo en los profesionales es una construcción tanto individual como social, a través de la cual se elabora la práctica y condiciona los tipos de relaciones que se establecen para el cuidado de la salud de los trabajadores. La percepción del concepto de salud en el trabajo es personal y subjetiva y puede comprenderse mejor contextualizada en el universo de creencias, valores y comportamientos del medio sociocultural. En el marco cultural de las representaciones, roles y expectativas, los profesionales configuran lo que cada uno entiende y vive como la salud en el trabajo y sobre ese significado atribuido resuelven cotidianamente cómo cuidar la salud de los trabajadores y cómo recuperarla cuando se ha deteriorado, siendo un proceso dinámico no sólo desde la propia experiencia personal sino también en relación con el contexto en que se desenvuelve.

De ahí que el concepto de salud en el trabajo implique una serie de significados para los profesionales en proceso de formación que ellos utilizan para orientar, interactuar, discutir, definir, categorizar e interpretar su comportamiento laboral y social. Sin embargo, no se conocen estudios publicados que describan los significados de la salud en el trabajo en los profesionales de la salud ocupacional. Por la importancia que tiene, en este estudio se planteó como objetivo general el explorar los significados culturales del concepto de salud en el trabajo en profesionales, tanto antes como después de la implementación de un evento específico de formación de recursos humanos, con el propósito de aportar datos útiles para el diseño curricular de nuevos programas de formación en salud ocupacional.

\section{MATERIAL Y MÉTODOS}

Tipo y diseño del estudio. Estudio de tipo cualitativo con diseño descriptivo exploratorio, realizado en dos fases y bajo el enfoque de la antropología cognitiva a través del modelo de dominios culturales. La delimitación espacio-temporal comprendió la ciudad de Guadalajara, México, de febrero a julio de 2011.

Población de estudio. Participaron los 18 estudiantes del diplomado en salud, seguridad en el trabajo y protección al ambiente, en fases sucesivas antes y después de la implementación del evento de formación. El tamaño de la muestra se basó en el supuesto del modelo de consenso cultural $^{21}$ para el estudio de patrones culturales. El supuesto plantea que en los estudios de descripción cultural, el tamaño de la muestra no debe ser grande, debido a que la correlación promedio entre informantes tiende a ser alta. Weller y Romney ${ }^{22}$ indican un tamaño mínimo de 17 informantes. Por ser la muestra mayor a ese número se consideró que se cumplió con el criterio de suficiencia y de saturación de la información.

Los 18 alumnos participantes estuvieron distribuidos en igual proporción de acuerdo con el género masculino o femenino. El promedio de edad fue de 35.83 años. Las profesiones que más sobresalieron entre los participantes fueron médicos y psicólogos. Habiendo solamente un ingeniero biomédico, ingeniero industrial, ingeniero químico, químico, trabajadora social y técnico ambiental. Entre los varones la profesión de médico fue mayor. En el género femenino la mayor la profesión encontrada fue psicología. El empleo de los alumnos fue diverso, sobresaliendo el trabajo de médico de empresa, supervisor de seguridad, supervisor de calidad y docentes. En menor medida se encontraron un administrativo y un auxiliar de investigación en institución educativa, un estudiante de doctorado, un psicólogo de empresa, un trabajador independiente y otra persona laborando en una asociación civil, así como dos participantes desempleados (11.11\%). 
Procedimiento y técnicas de recolección. La recolección sistemática de los datos en ambas fases se realizó a través de la técnica de listados libres ${ }^{23}$. La técnica consistió en pedir a los informantes un listado escrito de las cinco primeras palabras que les vinieran a la mente con el término salud en el trabajo. Posteriormente, se les indicó que explicaran sus respuestas. Dicha técnica sirvió para crear las listas y conocer los significados del concepto de salud en el trabajo a través de los elementos más frecuentes e importantes.

En ambas fases el tiempo de aplicación de la técnica no pasó de 10 minutos. En la primera fase el llenado de las listas libres se realizó el primer día del diplomado antes de cualquier actividad de enseñanza-aprendizaje. En la segunda fase las listas libres se llenaron el último día de clases antes de la ceremonia de clausura. En ambos casos, el llenado se realizó al mismo tiempo para todos los alumnos, dentro del aula de clases.

Análisis de los datos. Se llevó a cabo el análisis de dominios culturales, el cual tiene como objetivos obtener, desde los individuos, los términos que ellos usan para describir algún campo del conocimiento o dominio cultural e intentar descubrir los significados a través de las similitudes y las diferencias de esos términos.

El análisis de los listados se basó en tres supuestos: algunos términos o elementos del dominio son más sobresalientes, mejor conocidos, importantes o familiares que otros y cuando la gente hace listados libres tienden a mencionarlos en primer término; los individuos que poseen un mayor conocimiento del dominio listan más que aquellos que cuentan con un conocimiento menor, y los términos que más se mencionan indican los elementos más destacados de la temática ${ }^{23}$.

Los elementos de las listas se tabularon literalmente y se procesaron en el programa Visual Anthropac 1.0 - Freelists. Se obtuvo la clasificación de las palabras de acuerdo con la frecuencia de mención y con la relevancia a partir de la posición promedio de cada palabra en las listas. A partir de esos datos, los elementos se ordenaron a través de dos tablas para cada fase del estudio, una para los elementos destacados y otra para los elementos relevantes.

Para el ordenamiento de los elementos destacados, las palabras, de acuerdo a su frecuencia de mención, se clasificaron en elementos centrales, intermedios o periféricos. Los resultados obtenidos se interpretaron tratando de buscar los significados del concepto de salud en el trabajo, a partir de la definición, prácticas, componentes y atributos mencionados por los informantes.

Para la ordenación de los lexemas relevantes se obtuvo el producto del rango de cada elemento con su posición promedio en las listas. Donde 1 fue el rango final o relevancia más alta ${ }^{23}$. A través de este análisis se encontraron nuevos puntos de vista que no se observaron en la organización por frecuencias, sobre todo cuando algunos elementos se situaron al principio o al final de las listas.

Normas éticas. Por tratarse de un estudio realizado con seres humanos, se cumplieron las normas éticas del Comité de Investigación de la Declaración de Helsinki ${ }^{24}$. Localmente, la investigación se sujetó al Reglamento de la Ley General de Salud en Materia de Investigación de los Estados Unidos Mexicanos ${ }^{25}$, considerándose como una investigación sin riesgo. Finalmente, el Comité Científico y de Ética del Instituto de Investigación en Salud Ocupacional del Departamento de Salud Pública del Centro Universitario de Ciencias de la Salud de la Universidad de Guadalajara revisó el estudio y, en su momento, lo aprobó. Las personas entrevistadas conocieron los objetivos del estudio y autorizaron su participación voluntaria a través del consentimiento informado verbal. Se les garantizó la confidencialidad y el anonimato en el tratamiento de la información. Aclarando cualquier duda que surgió acerca de los procedimientos, riesgos y beneficios o cualquier otro asunto relacionado con la investigación y con datos personales. Así mismo, se les indicó que podían abandonar el estudio en el momento que quisieran. Sin embargo, todas las personas abordadas decidieron participar en el proceso completo. 


\section{RESULTADOS}

\section{Definición del concepto salud en el trabajo}

Los elementos del dominio de la salud en el trabajo, mencionados por los informantes, se encuentran en las tablas 1 y 2 , clasificados en primera y segunda fase, de acuerdo a la frecuencia de mención y a la relevancia, respectivamente.

En la primera fase del estudio, el concepto salud en el trabajo se definió a través de los elementos riesgo y seguridad. Los informantes revelaron: "Todos los trabajos implican riesgos físicos y mentales; éstos deben medirse o reconocerse antes de que exista un accidente laboral; es necesario conocer los riesgos a los que está expuesta la salud en el trabajo dentro de una empresa. La seguridad brinda estabilidad y salud; un ambiente seguro evita cualquier incidente o accidente de trabajo; con la seguridad se genera un ambiente idóneo para trabajar; con ella se trabaja con agilidad y confianza". Véase la tabla 1.

Tabla I. Elementos destacados por frecuencia de mención en el concepto de salud en el trabajo en profesionales en formación

\begin{tabular}{|c|c|c|c|}
\hline \multicolumn{2}{|c|}{ Elemento / Frecuencia de mención \% } & \multirow{2}{*}{ Significado } & \multirow{2}{*}{ Clasificación } \\
\hline Fase 1 & Fase 2 & & \\
\hline $\begin{array}{c}\text { Riesgo } 44.4 \% \\
\text { Seguridad } 44.4 \%\end{array}$ & $\begin{array}{l}\text { Seguridad } 50 \% \\
\text { Prevención } 50 \%\end{array}$ & Definición & Elementos centrales \\
\hline $\begin{array}{c}\text { Prevención } 38.9 \% \\
\text { Salud } 27.8 \% \\
\text { Higiene } 22.2 \% \\
\text { Normas } 22.2 \%\end{array}$ & $\begin{array}{c}\text { Accidentes } 27.8 \% \\
\text { Riesgos } 27.8 \% \\
\text { Capacitación } 22.2 \% \\
\text { Salud } 22.2 \% \\
\text { Normatividad } 22.2 \% \\
\text { Higiene } 22.2 \% \\
\end{array}$ & Prácticas & \multirow[b]{2}{*}{ Elementos intermedios } \\
\hline $\begin{array}{c}\text { Accidentes } 16.7 \% \\
\text { Bienestar } 16.7 \% \\
\text { Equipo } 16.7 \% \\
\text { Ergonomia } 16.7 \% \\
\text { Incapacidades } 16.7 \% \\
\text { Trabajadores } 11.1 \%\end{array}$ & $\begin{array}{c}\text { Normas } 11.1 \% \\
\text { Equipo de protección } \\
\text { personal } 11.1 \% \\
\text { Diagnóstico } 11.1 \% \\
\text { Medio ambiente } 11.1 \% \\
\text { Ambiente laboral } 11.1 \% \\
\text { Bienestar } 11.1 \%\end{array}$ & Componentes & \\
\hline $\begin{array}{c}5.6 \% \text { cada uno: } \\
\text { Aptitud, Aspectos, Autoprotección, } \\
\text { Básica, Calidad, Campañas } \\
\text { de vacunación y ejercicio } \\
\text { Capacitación, Comunicación, } \\
\text { Conciencia, Conocimiento, Control, } \\
\text { Corrupción, Crecimiento, Derecho. } \\
\text { Diagnóstico, Educación. Eficacia, } \\
\text { Empresa, Enfermedad física y } \\
\text { psíquica, Estructuras, Factores } \\
\text { de riesgo, Factores psicosociales, } \\
\text { Formatos, Información, Inversión, } \\
\text { Medio ambiente, Obligación, } \\
\text { Organización. Preparación, } \\
\text { Productividad Reglamentos, } \\
\text { Rehabilitación, Satisfacción, } \\
\text { Seguimiento, Tranquilidad. } \\
\text { Traslado por STPS }\end{array}$ & $\begin{array}{c}\text { 5.6\% cada uno: } \\
\text { Calidad. Capacidad, } \\
\text { Conocimiento. } \\
\text { Consecuencias. Control. } \\
\text { Cultura en seguridad, } \\
\text { Desarrollo profesional, } \\
\text { Empresas, Equipo, } \\
\text { Ergonomía, Estilo de vida } \\
\text { Evaluación, Extintores, } \\
\text { Factores de riesgo, Factores } \\
\text { psicosociales, Herramientas, } \\
\text { Integridad. Laboral, Ley, } \\
\text { Mejora continua. Moobing. } \\
\text { Organización. Peligros. } \\
\text { Planeación, Productividad, } \\
\text { Promoción, Seguridad e } \\
\text { Higine, Señalamientos, } \\
\text { Simulacros, Trabajadores, } \\
\text { Trabajo, Tranquilidad }\end{array}$ & Atributos & Elementos periféricos \\
\hline
\end{tabular}

Fuente: Directa 
En la segunda fase del estudio el concepto de salud en el trabajo se definió a través de los elementos prevención y seguridad. Los informantes dijeron: "La prevención es el objetivo de la salud en el trabajo, porque mantiene y preserva la salud e integridad de los trabajadores, evita o reduce los riesgos de accidentes y enfermedades y promueve la salud. La seguridad es inberente a la salud en el trabajo". Véase la tabla I.

\section{Prácticas del concepto salud en el trabajo}

Las prácticas del concepto salud en el trabajo, en la primera fase, se definieron a través de los elementos prevención, salud, higiene y normas. Los informantes indicaron: "La salud debe estar preservada y cuidada en una institución. En un ambiente laboral con bigiene se corre menos riesgo de desarrollar enfermedad laboral y se realizan las actividades con mayor calidad; aunque a muchas empresas no les importa la higiene empresarial. Las normas implican todo lo que rige a una empresa; son lineamientos que definen el actuar del patrón, empresa y empleados; la salud en el trabajo depende del conocimiento y cumplimiento de las normas y procedimientos; conocer y seguir las normas garantiza seguridad".

Las prácticas del concepto salud en el trabajo, en la segunda fase resultaron integradas por los lexemas accidentes, riesgos, capacitación, salud, normatividad e higiene. Los informantes señalaron: "Los accidentes deben evitarse para un mejor desempeño en el trabajo; influyen en la salud de los trabajadores; son frecuentes cuando no se cumple con la norma; la salud ocupacional abarca el reducir las tasas de accidentabilidad. La capacitación apoya la presencia y constancia de seguridad, prevención, salud y mejora continua; permite mejorar la productividad; debe adaptarse a los cambios científicos; es indispensable para instruir a los trabajadores y para que tomen conciencia".

\section{Componentes del concepto salud en el trabajo}

Con los elementos de frecuencia intermedia tales como accidentes, bienestar, equipo, ergonomía, incapacidades y trabajadores, se describieron los componentes del concepto de salud en el trabajo de la primera fase del estudio. En general, los informantes revelaron que: "El bienestar es la salud físico-psicológica dentro del trabajo; implica el mejoramiento ambiental laboral de los beneficios económicos, sociales y de productividad; con personas sanas se realiza mejor el trabajo; el ser saludable da la sensación agradable de continuar con proyectos. Trabajando en equipo se logran mejores resultados. La ergonomía facilita el trabajo en un ambiente sano y seguro; las condiciones ergonómicas deben ser adecuadas para que el trabajador haga su trabajo sin sufrir lesiones; estamos acostumbrados a trabajar con herramientas, mobiliario o peso que no siempre es el adecuado. Las incapacidades resultan después de un accidente de trabajo; necesita conocerse todo lo que conlleva otorgar una incapacidad. Los trabajadores son las personas que más pueden tener problemas en un área laboral”.

Los componentes del término de salud en el trabajo en la segunda fase de la investigación quedaron integrados por los lexemas normas, equipo de protección personal, diagnóstico, medio ambiente, ambiente laboral y bienestar. Los informantes expresaron lo siguiente: "El equipo de protección personal es indispensable en cualquier actividad laboral; brinda la seguridad necesaria para cuidar la salud. El diagnóstico sirve para implementar medidas eficientes en la prevención de enfermedades ya que se debe partir de un correcto diagnóstico situacional; es indispensable para hacer adecuaciones y/o modificaciones en una empresa. Teniendo un medio ambiente seguro $y$ controlado disminuye los riesgos a la salud; SSYMA (seguridad, salud y medioambiente, promoción e investigación. El ambiente laboral tanto organizacional como en los espacios y con compañeros es saludable para extrapolarlo a la sociedad". 


\section{Atributos del concepto salud en el trabajo}

En ambos fases, las atribuciones al concepto de salud en el trabajo estuvieron integradas por múltiples términos periféricos que reflejaron nociones individuales del concepto estudiado. Los atributos al concepto de salud en el trabajo en la primera fase del estudio quedaron integrados por 36 elementos que fueron mencionados solamente una vez. La mayor parte de los lexemas atribuidos al concepto de salud en el trabajo fueron de tipo positivo, sobresaliendo los términos corrupción, enfermedad física y psíquica y factores de riesgo como elementos negativos (Véase la tabla I).

En la segunda fase del estudio los atributos del concepto de salud en el trabajo se conformaron por 34 elementos casi todos ellos de tipo positivo, solamente se mencionaron los términos negativos de consecuencias, factores de riesgo, moobing y peligros (Véase la tabla I).

\section{Elementos relevantes del concepto de salud en el trabajo}

Los elementos más relevantes del concepto salud en el trabajo en la primera fase de la investigación fueron riesgo y seguridad. Dichos términos además de tener el rango 1 por su mayor frecuencia de mención aparecieron en una posición promedio en las listas libres entre 2.25 y 2.38, respectivamente. Véase la tabla II.

Tabla II. Elementos relevantes en el concepto de salud en el trabajo en profesionales en formación

\begin{tabular}{|c|c|c|c|c|c|c|c|c|c|}
\hline \multicolumn{5}{|c|}{ Fase 1} & \multicolumn{5}{|c|}{ Fase 2} \\
\hline Elemento & $\begin{array}{c}\text { Frecuencia } \\
(\%)\end{array}$ & Rango & \begin{tabular}{|c|} 
Posición \\
promedio
\end{tabular} & Relevancia & Elemento & $\begin{array}{c}\text { Frecuencia } \\
\text { (\%) }\end{array}$ & Rango & $\begin{array}{c}\text { Posición } \\
\text { promedio }\end{array}$ & Relevancia \\
\hline Riesgo & 44.4 & 1 & 2.25 & 2.25 & Prevención & 50 & 1 & 2 & 2 \\
\hline Seguridad & 44.4 & 1 & 2.38 & 2.38 & Seguridad & 50 & 1 & 2.11 & 2.11 \\
\hline Prevención & 38.9 & 2 & 2.71 & 5.42 & Normas & 11.1 & 4 & 1 & 4 \\
\hline Salud & 27.8 & 3 & 2.2 & 6.6 & $\begin{array}{l}\text { Peligros. Trabajo. } \\
\text { Protección. Cultura en } \\
\text { seguridad }\end{array}$ & 5.6 & 5 & 1 & 5 \\
\hline Básica, Empresa. Factores de riesgo & 5.6 & 7 & 1 & 7 & Accidentes & 27.8 & 2 & 2.6 & 5.2 \\
\hline Accidentes. Bienestar & 16.7 & 5 & 2 & 10 & Riesgos & 27.8 & 2 & 2.8 & 5.6 \\
\hline Higiene & 22.2 & 4 & 3.25 & 13 & Capacitación. Salud & 22.2 & 3 & 3 & 9 \\
\hline $\begin{array}{l}\text { Campañas de vacunación y } \\
\text { ejercicio, Derecho, Factores } \\
\text { psicosociales, Información. } \\
\text { Rehabilitación. Tranquilidad }\end{array}$ & 5.6 & 7 & 2 & 14 & Higiene & 22.2 & 3 & 3.25 & 9.75 \\
\hline Normas & 22.2 & 4 & 3.75 & 15 & Bienestar & 11.1 & 4 & 2.5 & 10 \\
\hline Equipo & 16.7 & 5 & 3 & 15 & $\begin{array}{l}\text { Trabajadores, Promoción, } \\
\text { Capacidad. Ley, Evaluación }\end{array}$ & 5.6 & 5 & 2 & 10 \\
\hline Ergonomía & 16.7 & 5 & 3.33 & 16.65 & Normatividad & 22.2 & 3 & 3.5 & 10.5 \\
\hline Trabajadores & 11.1 & 6 & 3 & 18 & Ambiente laboral & 11.1 & 4 & 3 & 12 \\
\hline Incapacidades & 16.7 & 5 & 4 & 20 & $\begin{array}{l}\text { Equipo de protección } \\
\text { personal Diagnóstico. } \\
\text { Medio ambiente }\end{array}$ & 11.1 & 4 & 3.5 & 14 \\
\hline $\begin{array}{l}\text { Aspectos. Calidad, Control, Corrupción. } \\
\text { Formatos. Enfermedad física y } \\
\text { psíquica. Formatos. Obligación, } \\
\text { Organización, Satisfacción }\end{array}$ & 5.6 & 7 & 3 & 21 & $\begin{array}{l}\text { Planeación, Seguridad e } \\
\text { higiene Señalamientos. } \\
\text { Productividad, } \\
\text { Tranquilidad. Ergonomía }\end{array}$ & 5.6 & 5 & 3 & 15 \\
\hline $\begin{array}{l}\text { Conciencia. Crecimiento. } \\
\text { Diagnóstico. Educación. Eficacia, } \\
\text { Reglamentos. Seguimiento, } \\
\text { Traslado por STPS }\end{array}$ & 5.6 & 7 & 4 & 28 & $\begin{array}{l}\text { Organización. Rehabilitación. } \\
\text { Control, Desarrollo } \\
\text { profesional. Equipo, } \\
\text { Conocimiento. Estilo de vida. } \\
\text { Factores psicosociales }\end{array}$ & 5.6 & 5 & 4 & 20 \\
\hline $\begin{array}{l}\text { Aptitud. Autoprotección. } \\
\text { Capacitación. Comunicación, } \\
\text { Conocimiento, Estructuras, } \\
\text { Inversión. Medio ambiente. } \\
\text { Preparación Productividad }\end{array}$ & 5.6 & 7 & 5 & 35 & $\begin{array}{l}\text { Mobbing. Simulacros. } \\
\text { Empresas. Calidad. } \\
\text { Consecuencias. Mejora } \\
\text { continua. Integridad, } \\
\text { Herramientas, Laboral. } \\
\text { Extintores. Factores de riesgo }\end{array}$ & 5.6 & 5 & 5 & 25 \\
\hline
\end{tabular}

Fuente: Directa.

Sin embargo, los elementos: básica, empresa y factores de riesgo, mencionados en la primera fase, a pesar de tener el último rango por su frecuencia de mención periférica, mostraron la primera posición promedio en las listas libres. Los informantes describieron 
estos términos de la siguiente manera: "La salud en el trabajo es básica para la realización del trabajo; está intimamente relacionada con el sector empresarial. Es indispensable conocer los factores de riesgo para educar y prevenir".

En la segunda fase del estudio los elementos más relevantes fueron prevención y seguridad. Tal como en la primera fase, estos lexemas obtuvieron el rango 1 por su mayor frecuencia de mención, y aparecieron en una posición promedio en las listas libres entre 2 y 2.11 , respectivamente. Véase la tabla 2. Los elementos que se colocaron en la primera posición promedio en las listas, a pesar de tener rangos finales por frecuencia de mención, fueron normas, peligros, trabajo, protección y cultura en seguridad. Los informantes refirieron estos términos así: "Toda actividad implica peligros de diferente magnitud. El desarrollo personal depende mucho de nuestro trabajo. Para lograr preservar la salud en el trabajo se debe proteger. La cultura determina las relaciones, actividades ypensamientos, en torno a un eje central, en este caso la seguridad".

\section{DISCUSIÓN Y CONCLUSIONES}

Los resultados, en ambas fases del estudio, muestran en el concepto de salud en el trabajo una visión completa de todos los elementos que lo integran, tal como lo expresan las principales organizaciones internacionales ${ }^{15-17}$. Los elementos más importantes del término se encuentran, antes del periodo de formación, en el riesgo y la seguridad, semejante a lo señalado por otros autores ${ }^{16}$. Después del periodo de formación, los elementos más importantes se hallan en la seguridad y la prevención, afín con la propuesta de la Unión Europea a través de la Estrategia Comunitaria de Salud y Seguridad en el Trabajo 2002-2006.

Antes del inicio del diplomado se destacó la intervención de las empresas y el conocimiento de los factores de riesgo como elementos básicos para la prevención. Al final del diplomado se destacó la cultura en seguridad y el conocimiento, aplicación y seguimiento de las normas para la preservación de la salud en el trabajo.

Con el estudio se refuerza el carácter cultural del término salud en el trabajo, sumándose a la evidencia de otros autores respecto de los conceptos de tutoría académica, atención en salud, salud, enfermedad y muerte, bienestar, no consumo de drogas y factores humanos causantes de accidentes ${ }^{6-14}$. La investigación realizada indica que los sistemas de significados que moldean la experiencia profesional, condicionan las percepciones e interpretaciones e influyen en la práctica profesional, pueden ser modificados a través de la formación.

Se concluye que los elementos para considerar en los programas de formación en salud ocupacional serían el partir del conocimiento de los riesgos y la normatividad como base para la construcción de la cultura de la prevención. Enfatizando que la cultura de la prevención es posible como el resultado de un proceso de aprendizaje. Para futuros estudios se sugiere explorar los significados del término de cultura de la prevención en los formadores de recursos humanos en salud en el trabajo.

\section{REFERENCIAS BIBLIOGRÁFICAS}

1. OMS. Estrategia mundial de la salud ocupacional para todos. Bol. Oficina Sanit Panam 1995; 119 (5): $442-$ 450. Disponible en la World Wide Web: <http://hist.library.paho.org/Spanish/BOL/v119n5p442.pdf> [Consulta: nov. 2011].

2. OPS. Plan Regional de Salud de los Trabajadores. Organización Panamericana de la Salud, División de Salud y Ambiente. Organización Mundial de la Salud. Washington. 1999. Disponible en la Word Wide Web: <http://www.who.int/occupational_health/regions/en/oehamplanreg.pdf> [Consulta: nov. 2011].

3. WHA. Salud de los trabajadores: plan de acción mundial. 60a Asamblea Mundial de la Salud. Washington. 2007. Disponible en la World Wide Web: <http://www.bvsde.paho.org/bvsast/fulltext/planesp.pdf> [Consulta: nov. 2011]. 
4. Luis Díaz, María Añez. Competencias y organización en Venezuela de los servicios de salud y seguridad en el trabajo. Gaceta laboral. 2009: 15 (3): 74-92. Disponible en la World Wide Web: <http://www.scielo. org.ve/pdf/gl/v15n3/art04.pdf $>$ [Consulta: dic. 2011].

5. Bernardo Restrepo. Investigación en Educación. Colombia. Instituto Colombiano para el fomento de la Educación Superior ICFES; 2002.

6. María de los Ángeles Aguilera, Teresa Torres, Martín Acosta, Elizabeth Pozos. Dimensiones culturales de la noción de tutoría académica en docentes y alumnos de doctorado en México. e-Gnosis Revista digital científica y tecnológica [online] 2011, no. 4. Disponible en la World Wide Web: <http://www.e-acervos. udg.mx/poeUDG/servlet/poe.GUIproducto/EGNOSIS/ES> [Consulta: oct. 2011].

7. Ana Alarcón, Aldo Vidal, Jaime Neira. Salud intercultural: elementos para la construcción de sus bases conceptuales. Rev Med de Chile 2003; 131 (9): 1061-1065. Disponible en la World Wide Web: <http:// www.scielo.cl/scielo.php?pid=S0034-98872003000900014\&script=sci_arttext $>$ [Consulta: dic. 2010].

8. Rodrigo Flores-Guerrero. Salud, enfermedad y muerte: lecturas desde la antropología sociocultural. Rev Mad. 2004; 10: 1-8. Disponible en la World Wide Web: <http://medicina.udea.edu.co/Dependencias/ Salud_y_Sociedad/paginaweb/Documentos\%20001/RodFlorez.pdf> [Consulta: nov. 2010].

9. Teresa Torres, Jazmín Munguía, Blanca Pozos, María de los Ángeles Aguilera. Representaciones sociales sobre la salud y la enfermedad de la población adulta de Guadalajara, México. Aten. Primaria 2010; 42 (3): 154-161. España. Disponible en la World Wide Web: <http://dialnet.unirioja.es/servlet/ articulo?codigo $=3171649>$ [Consulta: ene. 2011].

10. Ana Alarcón, Aldo Vidal. Dimensiones culturales en el proceso de atención primaria infantil: perspectivas de las madres. Salud Pública de México 2005; 47 (6): 440-446. México. Disponible en la World Wide Web: <http://www.scielosp.org/pdf/spm/v47n6/a08v47n6.pdf> [Consulta: nov. 2010].

11. Teresa Torres, Carolina Aranda, Manuel Pando, José Salazar. Dimensiones conceptuales del bienestar de personas con padecimientos crónicos. Revista de Salud Pública y Nutrición RESPYN [online] $2007 ; 8$ (4). México. Disponible en la World Wide Web: <http://www.respyn.uanl.mx/viii/4/articulos/bienestar.htm> [Consulta: ene. 2009].

12. María de los Ángeles Aguilera, Teresa Torres, Rosa Rodríguez y Martín Acosta. Dimensiones culturales del concepto de salud en trabajadores jubilados de Guadalajara, México. Hacia la Promoción de la Salud 2010; 15 (2): 13-27. Colombia. Disponible en la World Wide Web: <http://promocionsalud.ucaldas.edu.co/ downloads/Revista15(2)_2.pdf> [Consulta: dic. 2010].

13. Rubén Soltero. Perspectivas para el no consumo de drogas. En el libro Estudios sobre niñez y juventud. Estudios cualitativos. Ediciones de la Noche 2010. México.

14. Rosa Reyes, Lilia Prado, María de los Ángeles Aguilera, Rubén Soltero. Descripción de los conocimientos sobre factores humanos que causan accidentes en una industria arnesera mexicana. e-Gnosis Revista digital científica y tecnológica 2011; (5). México. Disponible en la World Wide Web: <http://www.eacervos.udg.mx/poeUDG/servlet/poe.GUIproducto/EGNOSIS/ES> [Consulta: oct. 2011].

15. Organización Internacional del Trabajo OIT. Enciclopedia de salud y seguridad en el trabajo. OIT-INSHT 1998; $3^{a}$ edición. España. Disponible en la World Wide Web: <http://www.ucm.es/info/seas/estres_lab/ enciclo/indice_gral.htm> [Consulta: nov. 2011].

16. Fernando García, Carlos Ruiz, Ana García. Salud laboral: conceptos y técnicas para la prevención de riesgos laborales. Editorial Masson 2000. Barcelona.

17. CISO e ICOH. Código internacional de ética para los profesionales de la salud ocupacional. ICOH CIST 2002. Italia. Disponible en la World Wide Web: <http://www.icohweb.org/core_docs/code_ethics_ spanish.pdf $>$ [Consulta: nov. 2011]

18. Unión Europea. Estrategia comunitaria de salud y seguridad en el trabajo 2002-2006. Síntesis de la legislación de la Unión Europea. Europa. Disponible en la World Wide Web: <http://europa.eu/legislation_ summaries/employment_and_social_policy/health_hygiene_safety_at_work/c11147_es.htm> [Consulta: dic. 2011].

19. Departamento de Seguridad y Salud Laboral Docente. Cultura de la prevención: la del compromiso por la seguridad y la promoción de la salud. Cultura Preventiva, Junta de Andalucía 2006; 1-5. España. Disponible en la World Wide Web: <http://www.juntadeandalucia.es/educacion/portal/com/bin/salud/ contenidos/BibliotecaVirtual/Articulos/CulturaPrevencion/1148387063088_cultura_de_la_prevencion-_ la_del_compromiso_y_la_participacion.pdf> [Consulta: nov. 2011].

20. Carolina Gómez. Salud laboral: una revisión a la luz de las nuevas condiciones de trabajo. Universitas psychologica 2007; 6 (1): 105-113. Colombia. Disponible en la World Wide Web: <http://sparta.javeriana. edu.co/psicologia/publicaciones/actualizarrevista/archivos/V6N109.pdf> [Consulta: nov. 2011].

21. Kimball Romney, Susan Weller, William Batchelder. Culture as consensus: A theory of culture and informant accuracy. American Anthropologist 1986; (88): 313-338. Estados Unidos. 
22. Susan Weller, Kimball Romney. Systematic Data Collection. 1988. Newbury Park, Beverly Hills, London, New Delhi: Sage.

23. Jacques Chevalier. Listado Libre. Sistemas de Análisis Social. 2006. Canadá. Disponible en la World Wide Web: <http://www.sas2.net/documents/tools/allpurpose/freelisting_es.pdf > [Consulta: jun. 2010]

24. Declaración de Helsnky de la Asociación Médica Mundial. Principios éticos para las investigaciones médicas en seres humanos. 59a Asamblea General, Seúl, Corea 2008. Disponible en la World Wide Web: http://www.wma.net/es/30publications/10policies/b3/17c_es.pdf

25. Estados Unidos Mexicanos. Reglamento de la Ley General de Salud en Materia de Investigación para la Salud 1983. México.

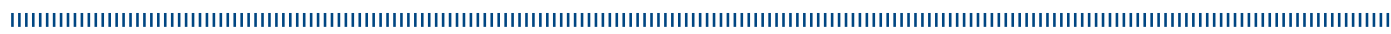

